\title{
Impact of ELM filaments on divertor heat flux dynamics in NSTX
}

\author{
J-W. Ahn*a , R. Maingi ${ }^{\mathrm{b}}$, J.M. Canik ${ }^{\mathrm{a}}$, K.F. Gan ${ }^{\mathrm{c}}$, T.K. Gray ${ }^{\mathrm{a}}$, and A.G. McLean ${ }^{\mathrm{d}}$ \\ ${ }^{a}$ Oak Ridge National Laboratory, Oak Ridge, USA \\ ${ }^{b}$ Princeton Plasma Physics Laboratory, Princeton, USA \\ ${ }^{c}$ Institute of Plasma Physics, Chinese Academy of Science, Hefei, China \\ ${ }^{d}$ Lawrence Livermore National Laboratory, Livermore, USA
}

\begin{abstract}
The ELM induced change in wetted area $\left(\mathrm{A}_{\text {wet }}\right)$ and peak heat flux $\left(\mathrm{q}_{\text {peak }}\right)$ of divertor heat flux is investigated as a function of the number of striations, which represent ELM filaments, observed in the heat flux profile in NSTX. More striations are found to lead to larger $\mathrm{A}_{\text {wet }}$ and lower $\mathrm{q}_{\text {peak }}$. The typical number of striations observed in NSTX is $0-9$, while $10-15$ striations are normally observed in other machines such as JET, and the ELM contracts heat flux profile when the number of striations is less than 3-4 but broadens it with more of them. The smaller number of striations in NSTX is attributed to the fact that NSTX ELMs are against kink/peeling boundary with lower toroidal mode number ( $\mathrm{n}=1-5)$, while typical peeling-ballooning ELMs have higher mode number of $n=10-20$. For ELMs with smaller number of striations, relative $A_{\text {wet }}$ change is rather constant and $\mathrm{q}_{\text {peak }}$ change rapidly increases with increasing ELM size, while $\mathrm{A}_{\text {wet }}$ change slightly increases leading to a weaker increase of $\mathrm{q}_{\text {peak }}$ change for ELMs with larger number of striations, both of which are unfavourable trend for the material integrity of divertor tiles.
\end{abstract}

PACS: 52.55.Fa, 52.55.Rk, 55.25.Fi

PSI-21 Keywords: Divertor heat flux, ELM, Filament, NSTX 
Corresponding Author Address: Princeton Plasma Physics Laboratory, Princeton, NJ 08543, USA

Corresponding Author e-mail: jahn@pppl.gov

Presenting Author: Joon-Wook Ahn

Presenting Author e-mail: jahn@pppl.gov

\section{Introduction}

Edge localized mode (ELM) is a serious threat to the material integrity in fusion devices because of its high heat and particle flux to the divertor surface, therefore needs to be controlled to avoid unacceptable damage to the divertor via appropriate ELM suppression or mitigation techniques. Since it is not yet clear if ITER would be able to achieve complete ELM suppression, it is important to accurately estimate the acceptable size of ELM for the range of key operating parameters, for example the plasma current $\left(\mathrm{I}_{\mathrm{p}}\right)$ [1]. In this estimation, the effective area of divertor surface onto which the ELM power is distributed is an important parameter. The total divertor area is fixed for a specific machine and therefore it's more beneficial for limiting the peak divertor heat flux if the total ELM power is dispersed on a higher fraction of the total surface area. This is equivalent to the broadening of divertor heat flux profile by ELMs and this effect is important to alleviate the requirement of ELM mitigation system performance. For example, JET shows profile broadening by ELMs all the time and the broadening becomes more effective for bigger (i.e. larger energy loss) ELMs [2]. Since an ELM event represents an unstable MHD mode with a finite toroidal mode number (n), and consists of multiple filamentary structures that detach from the plasma surface and carry high heat and particle fluxes along the 
magnetic field line to the divertor [3,4], the profile broadening is generally attributed to the heat flux carried by ELM filaments, which tend to disperse the overall ELM power over a large area at the divertor surface. These ELM filaments appear in the measured heat flux profile in the form of striations and represent local peaks or bumps in the 1-D radial profiles. The typical number of striations observed in JET is $\sim 15$ [5]. However, in NSTX, we normally observe $0-9$ striations and both the profile broadening and contraction by ELMs have been found. This is believed to be due to the fact that NSTX ELMs are at the peeling boundary that has lower toroidal mode number $(\mathrm{n}=1-5)[6,7]$, than the typical peeling-ballooning ELMs with higher mode number $(\mathrm{n}>10)[3,8]$. Following a brief description of diagnostics and the definition of relevant parameters, data for ELMs with various numbers of striations in NSTX will be presented, and the implication for ITER will be discussed.

\section{Experimental setup and data analysis procedure}

NSTX is equipped with a high speed infrared (IR) camera to monitor surface temperature with spatial resolution of $\sim 6 \mathrm{~mm}$ and frame rate $1.6-6.3 \mathrm{kHz}$. In order to take ELM data the camera was operated at $5-6 \mathrm{kHz}$, which can usually provide $2-3$ data points during the ELM rise phase. The temporal array of measured 2-D surface temperature is used in a 3-D heat conduction solver, TACO, to produce 2-D heat flux profiles. The original version of TACO has been improved to address the effect of thin hydrocarbon layer on the tile surface that forms naturally by the interaction of carbon tile with the plasma [9]. The heat transmission coefficient of the layer, $\alpha$, is introduced in the procedure of solving the heat conduction equation with values

of $30-60 \mathrm{kWm}^{-2} \mathrm{~K}^{-1}$ for the data analysis in this work. The calculated 2-D heat flux profiles are 
re-mapped from the $(\mathrm{x}, \mathrm{y})$ to the $(\mathrm{r}, \Phi)$ plane, which shows the toroidal and radial structure of heat flux deposition more clearly.

1-D radial heat flux profiles taken at multiple toroidal locations are averaged over to produce a mean 1-D radial profile, $\bar{q}(r)$, for the data analysis in order to represent the whole 2-D heat flux data observed by the IR camera. The total deposited power to the divertor surface can be obtained by integrating this radial profile in both radial and toroidal directions; $P_{d i v, I R}=$ $\int 2 \pi r \bar{q}(r) d r$. A characteristic area onto which the total power is deposited, assuming that it is all carried by the peak heat flux ( $\left(\mathrm{q}_{\text {peak }}\right)$, is defined as the wetted area $\left(A_{\text {wet }}=P_{\text {div,IR }} / q_{\text {peak }}\right)$. It is

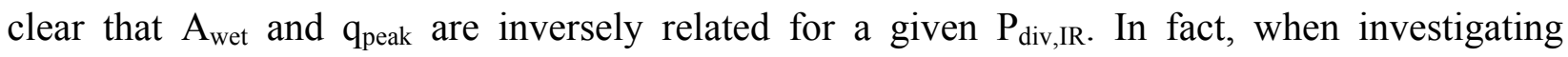
divertor heat flux by ELMs, $\mathrm{P}_{\mathrm{div}, \mathrm{IR}}$ is primarily determined by how much power is ejected from the plasma by the ELM and $q_{\text {peak }}$ determines the peak surface temperature $\left(T_{\text {peak }}\right)$ that must be maintained below a certain level (e.g., melting temperature of tile material) from the operations point of view. Therefore, increasing $\mathrm{A}_{\text {wet }}$ is necessary to keep $\mathrm{q}_{\text {peak }}$ as low as possible. Similarly, a characteristic length in the radial direction that represents how wide the heat flux profile is, i.e. integral heat flux width, can be defined; $\lambda_{q}=P_{d i v, I R} / 2 \pi r_{\text {peak }} q_{\text {peak }}=A_{\text {wet }} / 2 \pi r_{\text {peak }}$. Also obtained from the heat flux data is the total deposited energy to the divertor surface by time integrating deposited power; $W_{d i v, I R}=\int P_{d i v, I R} d t$. Temporal evolution of these parameters, particularly $\mathrm{A}_{\text {wet, }}, \mathrm{P}_{\mathrm{div}, \mathrm{IR}}$, and $\mathrm{q}_{\text {peak }}$, during the ELMs will be presented and the relation with the number of ELM filaments will be discussed in the remainder of this paper.

\section{Results}

3.1 Change of $\mathrm{A}_{\text {wet }}$ and $\mathrm{q}_{\text {peak }}$ with the number of ELM striations 
The number of striations caused by an ELM can be effectively counted by comparing both 1-D and 2-D heat flux profile data during the ELM to those taken immediately before the ELM. Temporal evolution of $\mathrm{A}_{\mathrm{wet}}, \mathrm{P}_{\mathrm{div}, \mathrm{IR}}$, and $\mathrm{q}_{\text {peak }}$ across an ELM, obtained from the procedure explained above, are shown in figure 1(a) for the case of one ELM striation. Radial heat flux profiles are shown in figure 1(b) for three time slices, and they are color coded for the three stages indicated by the vertical lines in figure 1(a); immediately before the ELM (red), during the ELM rise phase (blue), and at the ELM peak time (green). A local peak, indicated by the black arrow, is observed in the blue and green profiles and this is from the ELM filament. $A_{w e t}$ for the ELM rise phase $\left(=0.28 \mathrm{~m}^{2}\right.$, blue $)$ is found to decrease by $\sim 20 \%$ compared to the inter-ELM value $\left(=0.33 \mathrm{~m}^{2}\right.$, red) and it remains almost unchanged at the ELM peak time (green). Due to this $\mathrm{A}_{\text {wet }}$ reduction, $\mathrm{q}_{\text {peak }}$ increased by a factor $\sim 5.7\left(\mathrm{q}_{\mathrm{peak}}=1.28 \rightarrow 7.32 \mathrm{MW} / \mathrm{m}^{2}\right)$ although the power increase was only by a factor $\sim 4.6\left(\mathrm{P}_{\mathrm{div}, \mathrm{IR}}=0.42 \rightarrow 1.95 \mathrm{MW}\right)$. On the other hand, figure 2 shows the case of an ELM with 5 striations. It is clear from figure 2(a) that $\mathrm{A}_{\mathrm{wet}}$ increases, contrary to the data in figure 1(a), and this profile broadening helped reduce the divertor surface overheating ( $\mathrm{q}_{\text {peak }}$ increase by only a factor $\sim 2.7, \mathrm{q}_{\text {peak }}=0.71 \rightarrow 1.92 \mathrm{MW} / \mathrm{m}^{2}$, while the total power increase by the ELM was more than a factor $\left.\sim 3.6, \mathrm{P}_{\mathrm{div}, \mathrm{IR}}=0.32 \rightarrow 1.15 \mathrm{MW}\right)$. From the data in figures 1 and 2, we can see the beneficial effect of profile broadening and obtain an important implication that the number of striations appearing in the heat flux profile may be closely related to the broadening or contraction of the profile induced by ELMs. Observations in NSTX demonstrate that $\mathrm{A}_{\mathrm{wet}}$ decreases (i.e. the profile contracts) when the number of striations is less than $3-4$ and increases (profile broadening) when it is more. Because the striation number of $3-4$ is in the borderline, the profile either shows broadening or contraction, depending on the specific pattern of the spatial distribution of filament heat flux. 
Figure 3 is a summary of $\mathrm{A}_{\text {wet, }}, \mathrm{P}_{\text {div,IR }}$, and $\mathrm{q}_{\text {peak }}$ change as a function of the number of striations observed in the profile for many ELMs. Each data point was taken at the ELM peak time, i.e. when the total deposited power reached maximum, and there are two groups of data shown in the figure. The red points represent ELMs with $0-4$ striations (taken from plasmas with weaker shaping) and the blue points are for ELMs with $2-9$ striations (taken from strongly shaped plasmas). Figure 3(a) is a plot of $A_{w e t}$ change by ELMs, relative to the inter-ELM value, as a function of the number of observed striations. For the group of $0-4$ striations, profile contracts, i.e. $\Delta \mathrm{A}_{\mathrm{wet}}<0$, for most of the data, while the group of $2-9$ striations showed profile broadening, i.e. $\Delta \mathrm{A}_{\mathrm{wet}}>0$, for many of the data point. It is clear from this combined dataset that 3 - 4 striations are indeed in the borderline between the broadening and contraction. The size of ELMs, represented by the power increase by ELMs relative to the inter-ELM values, was rather uncorrelated with the number of striations for this dataset, see figure 3(b). Therefore, the increase of $A_{\text {wet }}$ change along with constant ELM size leads to the decrease of $\mathrm{q}_{\text {peak }}$ change as the number of striations becomes larger, see figure 3(c). This is consistent with the examples shown in figures 1 and 2.

3.2 Change of $A_{\text {wet }}$ and $q_{\text {peak }}$ with the ELM size

While ELM heat flux can pose a serious threat to the material integrity, one ameliorating factor has been that $\mathrm{A}_{\text {wet }}$ has been observed to increase with the size of the ELM, as compared to the inter-ELM heat flux profile [2]. Therefore it is important to check the trend for the ELMs that show profile contraction reported in section 3.1. Figure 4 shows $\mathrm{q}_{\text {peak }}$ and $\mathrm{A}_{\text {wet }}$ measured at ELM peak times as a function of relative ELM power, again used as a metric of the ELM size. Figure 
4(a) shows that the size of relative $\Delta \mathrm{A}_{\text {wet }}$ for ELMs with $0-4$ striations is rather constant, while that for $2-9$ striations slightly increases, with the increase of ELM size. This $\Delta \mathrm{A}_{\text {wet }}$ change leads to a clear increase of $\Delta q_{\text {peak }}$ with the increasing ELM size for both dataset, shown in figure 4(b), and the increase is more rapid for the case of $0-4$ striations. This trend is opposed to that shown in JET [2], which demonstrated rather constant $q_{\text {peak }}$ due to increasing $A_{\text {wet, }}$ as the ELM crash becomes larger. The trend shown in figure 4 is clearly harmful to the integrity of divertor tiles and should be properly taken into account when determining the requirement of ELM mitigation system performance, if a similar trend is projected for a possible operating regime, in a future machine such as ITER.

\section{Discussion}

Striations in the heat flux profile represent ELM filaments and therefore are directly related to the toroidal mode number of ELMs. Assuming that the IR camera view covers sufficient radial range for all ELM filaments to be captured, we believe that the striation data of NSTX reported in this paper well represent the ELM filaments. In fact, we confirmed from a wide angle visible camera data that covers almost full toroidal and radial range of lower divertor [10], thanks to the compact geometry of spherical tokamak, that some ELM filaments occur more than once at different radial locations at one time slice. This is because an ELM filament follows the field line and helically deposits heat and particle flux to the divertor surface, and therefore a single striation can revolve toroidally more than once. This indicates that the toroidal mode number of ELM filaments can be smaller than the counted number of striations from the camera 
data, which is consistent with the quoted n-number of ELMs in NSTX $(n=1-5)[6,7]$ and the number of observed striations $(0-9)$ from the IR data reported in this work.

As discussed earlier, the peeling nature of ELMs are thought to be responsible for the lower n-number at NSTX, while typical peeling-ballooning ELMs have n-numbers higher than 10 as observed in other tokamaks such as MAST, JET, and ASDEX-U [3,4,5,8]. The relationship of ELM heat flux dynamics, particularly for the change of $A_{w e t}$ and $q_{p e a k}$, to the number of striations presented in this paper indicates that peeling-ballooning ELMs are more advantageous to achieve the broadening of heat flux profile, therefore to reduce the requirement of ELM mitigation system performance in the future machine. Since the stability of ITER pedestal is expected to be limited by current-driven kink/peeling modes due to its low collisionality and modestly strong shaping [11], detailed study of projected profiles and stability analysis for ITER are expected to be essential for more accurate prediction.

\section{Acknowledgments}

This research was supported by the U.S. Department of Energy, contract numbers DE-AC05-00OR22725 (ORNL) and DE-AC02-09CH11466 (PPPL).

\section{Reference}

[1] A. Loarte et al., Nucl. Fusion 54 (2014) 033007

[2] T. Eich et al., J. Nucl. Mater. 415 (2011) S856

[3] A. Kirk et al., Phys. Rev. Lett. 92 (2004) 245002

[4] A. Kirk et al., Phys. Rev. Lett. 96 (2006) 185001

[5] S. Devaux et al., J. Nucl. Mater. 415 (2011) S865 
[6] R. Maingi et al., Phys. Rev. Lett. 103 (2009) 075001

[7] D. P. Boyle et al., Plasma Phys. Control. Fusion 53 (2011) 105011

[8] T. Eich et al., Phys. Rev. Lett. 91 (2003) 195003

[9] K. F. Gan et al., Rev. Sci. Instrum. 84 (2013) 023505

[10] F. Scotti et al., Rev. Sci. Instrum. 83 (2012) 10E532

[11] P.B. Snyder et al., Nucl. Fusion 51 (2011) 103016 


\section{Figure captions}

Figure 1 Temporal evolution of $\mathrm{A}_{\text {wet }}, \mathrm{P}_{\mathrm{div}, \mathrm{IR}}$, and $\mathrm{q}_{\text {peak }}$ (a) and 1-D radial heat flux profiles (b), for an ELM with 1 striation, indicated by a black arrow, during the ELM rise phase and at the ELM peak time. Reduction of $A_{\text {wet }}$ by $\sim 20 \%$ by the ELM is seen in plot (a). Heat flux profiles are color coded for specific time slices indicated by the vertical lines in the left plot.

Figure 2 Temporal evolution of $A_{\text {wet }}, P_{\text {div,IR }}$, and $q_{\text {peak }}$ (a) and 1-D radial heat flux profiles (b), for an ELM with 5 striations, indicated by black arrows, at the ELM peak time. $A_{\text {wet }}$ increase by the ELM is clear in plot (a). Heat flux profiles are color coded for specific time slices indicated by the vertical lines in the left plot.

Figure 3 Change of $A_{w e t}(a), P_{\text {div,IR }}(b)$, and $q_{\text {peak }}$ (c) by ELMs, relative to the value immediately before the ELM, as a function of the number of striations observed in the heat flux profile. The red points represent a group of ELMs with $0-4$ striations observed (weaker plasma shape), and the blue points are for the ELM group with $2-9$ striations (stronger plasma shape).

Figure 4 Relative change of $A_{\text {wet }}$ and $\mathrm{q}_{\text {peak }}$ measured at ELM peak times, for a group of ELMs with $0-4$ striations (same as in figure 3 , red points) and $2-9$ striations (blue points) as a function of ELM size. ELM power divided by the inter-ELM power was used as a metric of the ELM size. 
Figures

Figure 1:
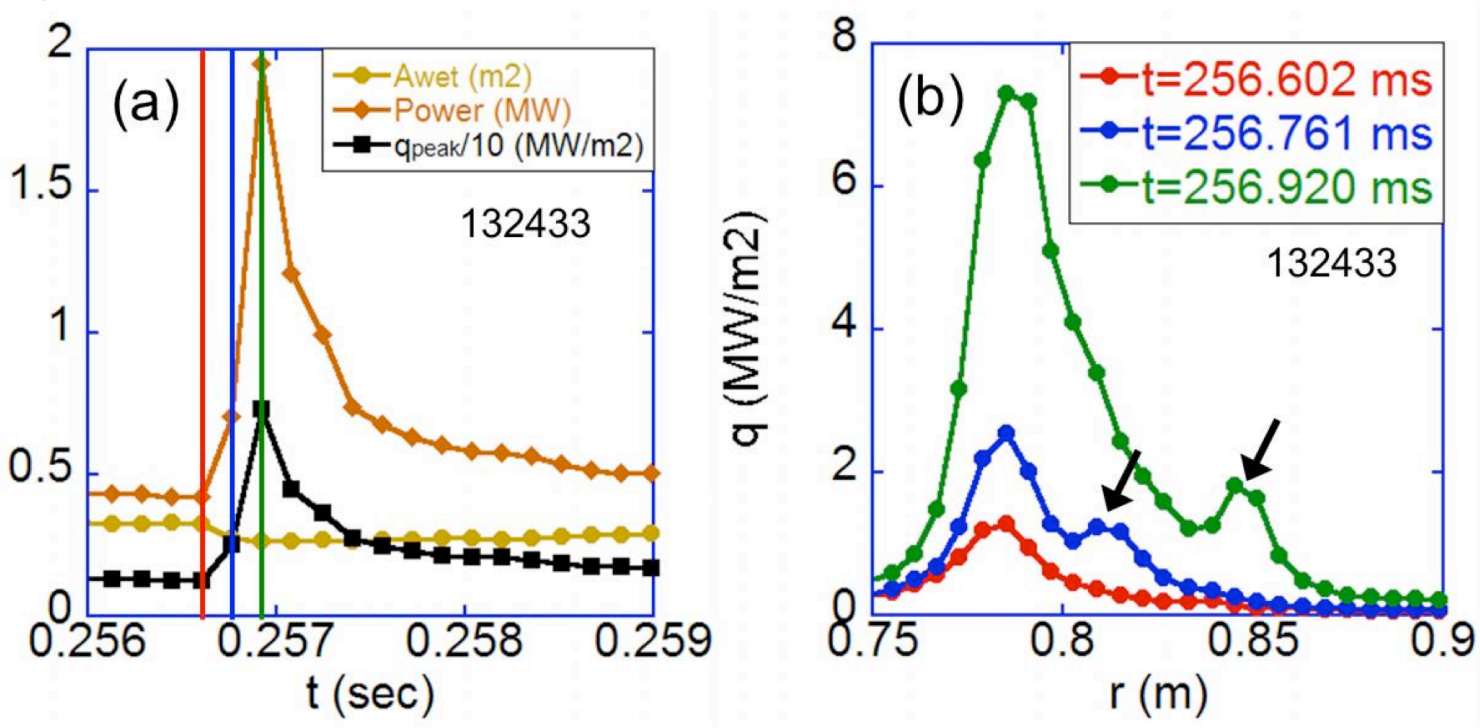
Figure 2:

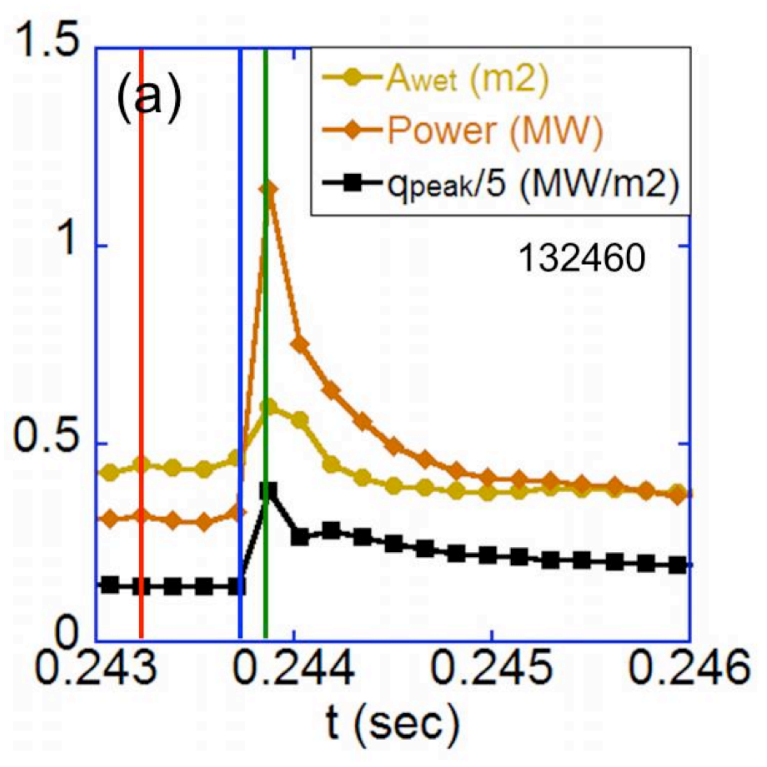

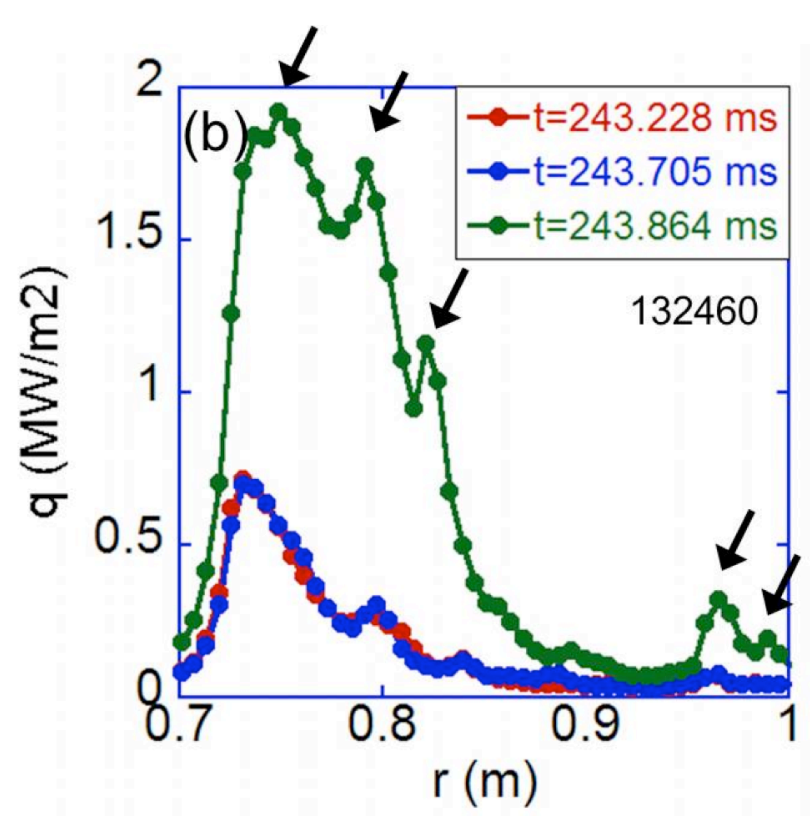


Figure 3:
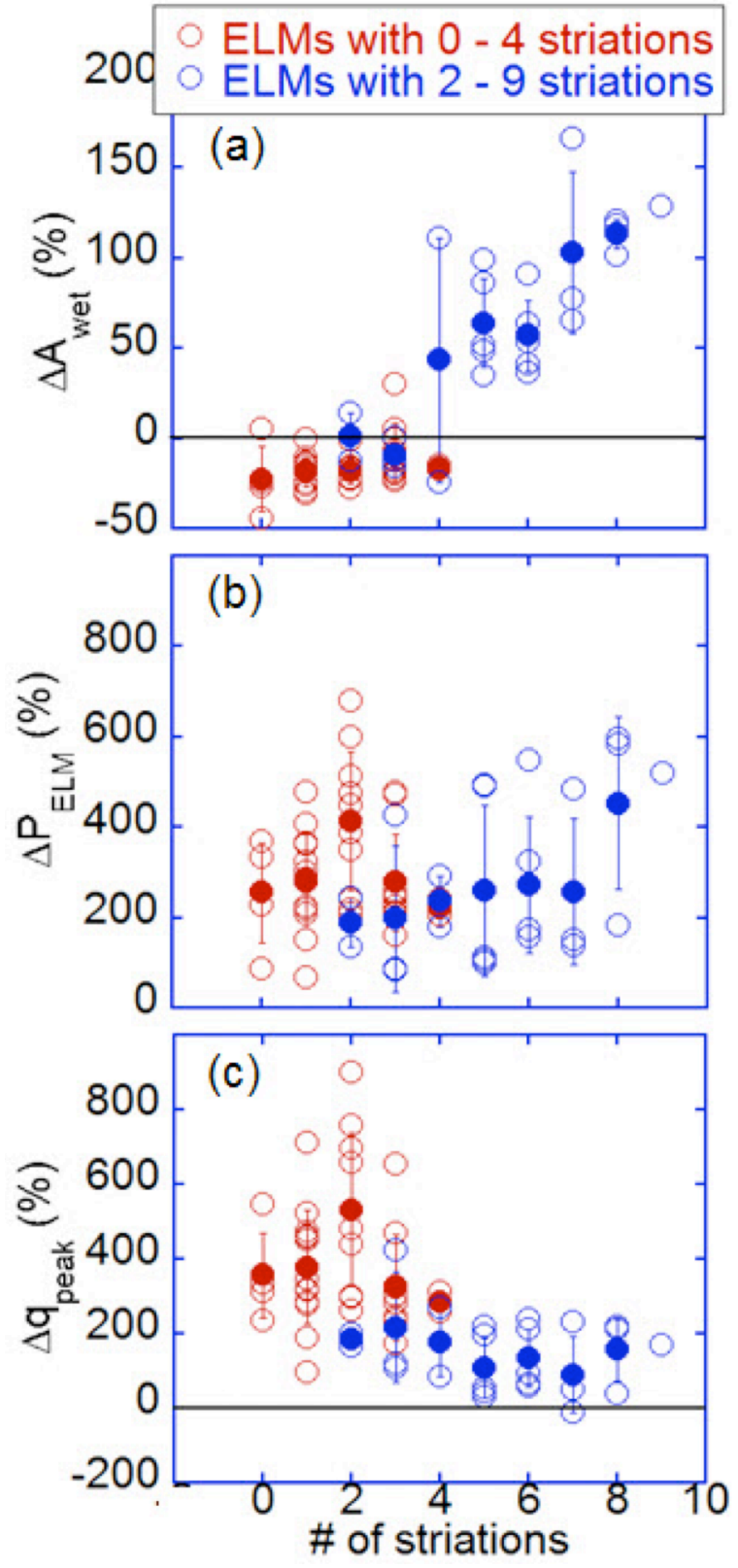
Figure 4:
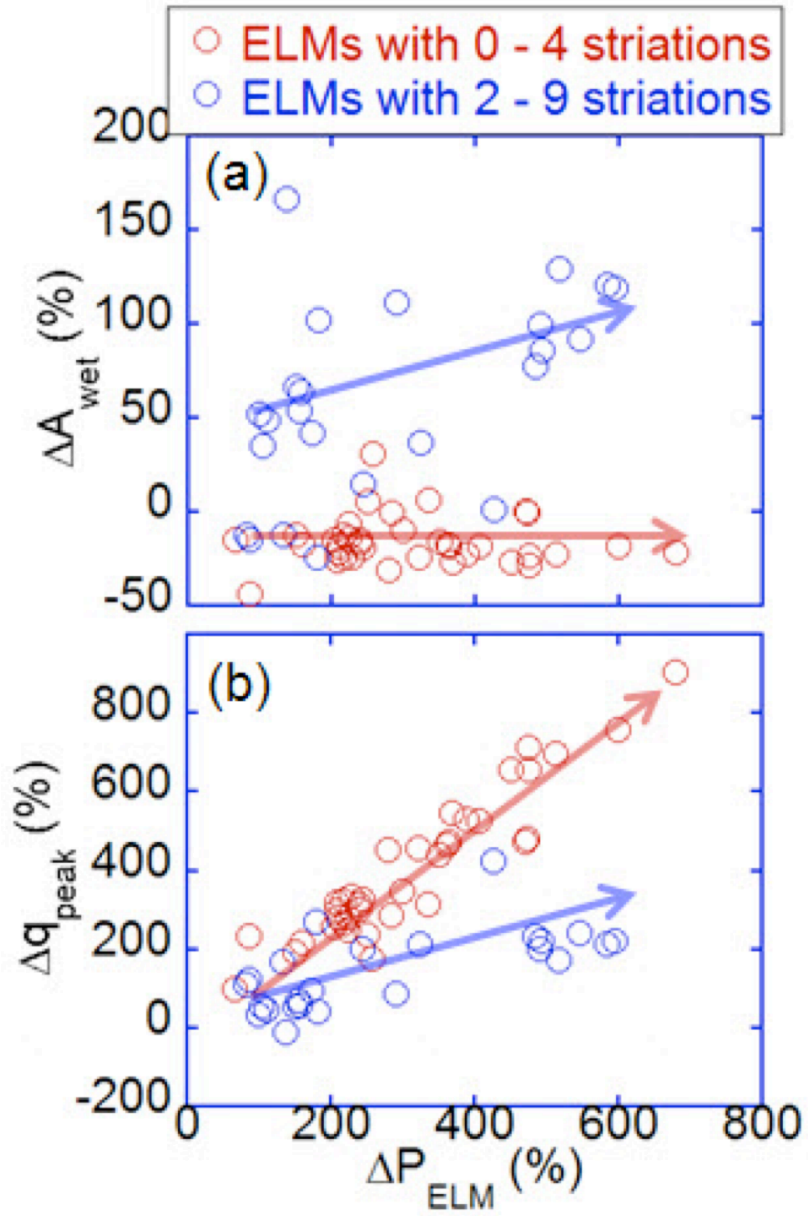